\title{
Quantized Charges and Currents in the Electromagnetic Field
}

\author{
Jacques Consiglio $^{1}$ \\ ${ }^{1}$ 52, Chemin de Labarthe, Labastidette, France \\ Correspondence: Jacques Consiglio, 52, Chemin de Labarthe, Labastidette F31600, France. Tel: 33-687-493-664. \\ E-mail: Jacques.Consiglio@gmail.com
}

Received: November 2, 2012 Accepted: December 22, 2012 Online Published: January 16, 2013

doi:10.5539/apr.v5n1p126

URL: http://dx.doi.org/10.5539/apr.v5n1p126

\begin{abstract}
We consider the demonstration of Dirac charge quantization and interpret the main equation as the signature of a current of magnetic poles building the electron wave and mass. This naturally leads to opposite coefficients in Dirac's equation, and to series of small quanta of magnetic charge $g=a e / 2 n$ including one in exact agreement with Mikhailov experimental estimate $g=a e / 6$. The resulting theoretical monopole wave is a quantized current of electricity that cancels or replaces the Dirac string. Energy is the effect of the magnetic current interacting with an electric charge, and the theory trivially agrees with the energy gap of hydrogen orbitals $E=E_{0} / n^{2}$. A second series of charges $g=n a$ e comes out of the equations, which it is shown compatible with the electrons, muons and tauons mass built from a finite suite of magnetic poles. Once opposite coefficients are taken into account, the symmetry of magnetism and electricity discovered by Dirac matches with experimental charges and involves charges and currents.
\end{abstract}

Keywords: magnetic monopole, Dirac quantization, Mikhaïlov charge, energy quantization, origin of mass, fine structure constant.

\section{Introduction}

Magnetic poles were considered by Maxwell (1873), Curie (1894) and a minimal charge originally predicted by Dirac (1931) in a famous paper $\left(g_{D}=e / 2 \alpha\right)$. All other known theories predict either the same charge or the double. But Ehrenhaft (1930, 1944), Schedling (1950) and Ferber (1950) measured magnetic charges in disagreement with Dirac's prediction; more recently, but still more than 20 years ago, Mikhaillov (1982, 1985, $1987,1991)$ had similar results and estimated the magnetic quantum of charge to $g_{M}=\alpha e / 6$, about 50,000 times smaller than predicted; some other authors consider an experimental quantum $g=\alpha e$. But Mikhaïlov (1991) also found charges compatible with Dirac prediction in a Millikan-like experiment with ferromagnetic particles in water droplets. Thus one of the mysteries of modern physics to which no theoretical solution seems to exist.

Repeated experimental results suggest that the theory is incomplete. So, we interpret the main equation of Dirac's (1931) paper in a different direction related to our previous publications (Consiglio, 2012a, 2012b), in which particles mass and wave result from their interaction with magnetic poles, and a magnetic current or monopoles flux is its origin. The object of the present paper is to push the analysis in a more conventional direction which addresses the relations between the electron charge, the fine structure constant, and the magnetic monopole charge. This theory, although it can be seen as contradicting Dirac quantization, is not foreign to his ideas and equations but solely uses a complimentary definition of the wave. It will show that the connection between electric and magnetic charges found by Dirac is true, but that the coefficients in the equation are not appropriate, and that experimentally measured charges, including Mikhaïlov's, are relevant in quantum physics and in agreement with our assumption on the nature of the wave.

Concerning the structuring constants and their relations with elementary charges, it will uncover a general symmetry of electromagnetism governing the numerical ratio between particles charge and their wave which we find in connection with the so called Dirac string.

The trivial substitutions we propose in a few classical equations provide a very simple understanding of some fundamental mysteries of quantum physics.

In this paper, we use classical electromagnetism and early quantum physics concepts to the maximum extent, because if our logic and deductions are correct, further developments of physics miss a fundamental point, which is addressed in the form of mathematical abstractions hiding the underlying phenomena. 


\section{General Aspects}

The main object of this section is to show how some of the ideas in this paper initially came.

\subsection{Dirac Quantization}

In his celebrated demonstration of charge quantization, Dirac (1931) analyzes the continuity of an electron wave function around a field singularity, a singular line along which the wave function vanishes. The field of the monopole $\boldsymbol{H}$ is defined by a vector potential $\boldsymbol{A}$ such that: curl $\boldsymbol{A}=\mathrm{gr} / \mathrm{r}^{3}$. Using a wave function $\psi$ of the electron in the absence of external field, interpreted as a density of probability of presence, Dirac introduces a function $\exp (i \beta)$ which is the difference between a normal wave function $\psi$ in the absence of external fields and the wave function in the presence of external fields: $\psi \rightarrow \psi \exp (i \beta)$. Then he does his magic showing that the function $\beta$ has quite specific properties; it has no definite value at each point, it is not integrable, but it must have definite derivatives: $k_{i}=\delta \beta / \delta x_{i}$ with $x_{i}$ in $\{x, y, z, t\}$ which are proven independent of the chosen wave function $\psi$ and then interpreted as the effect of the "fields of forces"; since for the normal wave function $\psi$, the norm $|\psi|$ represents the current of probability of presence of the electron, the phase variation $\beta$ gives the effects of the fields on this current, and then according to Dirac (1931, Sect. 2, Eq. 7):

$$
\operatorname{curl}(\boldsymbol{k})=(e / \mathrm{hc}) \boldsymbol{H} ; \quad \operatorname{grad}\left(k_{4}\right)-\partial \boldsymbol{\kappa} / \partial t=(e / /) \boldsymbol{E}
$$

The change in phase of the electron wave around a closed curve is then $\int(k, d \boldsymbol{l})=\int(\operatorname{curl}(k), d \boldsymbol{S})$, according to Stoke's theorem ( $d \boldsymbol{l}$ is a line element of the closed curve and $d \boldsymbol{S}$ is an element of a surface bounded by the closed curve). A magnetic pole has no electric field, and assuming continuity of the electron phase around the singular line naturally leads to (Dirac, 1931, Sect. 3, Eq. 8 - 9):

$$
2 \pi n=(e / h c) \int(\boldsymbol{H}, d \boldsymbol{S})=4 \pi e g / h c
$$

This is the famous Dirac quantization condition; it answers the question introduced in Dirac (1931) paper about the physical reason for $h c / e^{2}=137$, in the form of reciprocal quantization of electric and magnetic charges.

Conceptually, the demonstration leads to two remarks: First, Dirac considers implicitly the electron wave to be passive. Numerous quantum experiments show on the contrary that the wave can be considered active, in the manner of the theories of de Broglie (1927) and Vigier-Bohm (1954). The underlying physics could then be different, in particular: i) if the electron wave is strongly energetic, but this raises the question of the energy source; ii) the wave could be discrete or have a small granularity; iii) if the wave is a current, the interpretation of the wave function as a density of probability of presence is incomplete and the logic partly irrelevant.

The second remark concerns the singular line, the so called Dirac string, which is sometimes considered as an artifact of the mathematical representation; but the singularity is the central concept of Dirac's demonstration, and it is not physical; at least not trivially. Then possibly something physical is missing and cancels the string.

\subsection{A Different Symmetry}

Denoting $g_{D}$ the minimal Dirac charge, from (2) we have $h c / e=2 g_{D}$, and then substituting in (1):

$$
2 g_{D} \operatorname{curl}(\boldsymbol{k})=\boldsymbol{H} ; 2 g_{D}\left(\operatorname{grad}\left(k_{4}\right)-\partial \boldsymbol{\kappa} / \partial t\right)=c \boldsymbol{E}
$$

This is the same equation as (1), as we only changed the notations, and it does address the impact of a field on an electron wave in any situation and now it has a pure classical form with electric and magnetic charges.

But then what is a monopole charge doing in there? We first notice that Dirac charge is a characteristic of the electron wave, at least numerically. It is the coefficient to the second derivatives in (3), which can be seen as the signature of a magnetic current and field expressed in units of magnetic charge per meter per second.

The wave function of an electron in the absence of external fields is modified by the presence their presence in agreement with $\psi \rightarrow \psi \exp (i \beta)$. As we interpret the electron wave as a magnetic current corresponding to an oscillating magnetic field, taking simply $\psi=\exp (i \gamma)$, the wave function in the presence of an external field is $\exp (i(\gamma+\beta))$. Then we can apply Dirac's reasoning to $(\gamma+\beta)$ and identify the resulting $k_{i}=\delta(\gamma+\beta) / \delta x_{i}$ to a description of the total fields, including the electron's electric and magnetic fields and currents (its wave). But now the $k_{i}$ include the electron's phase and (3) rules its variations.

Now looking at classical equations, and using Faraday's law of induction, denoting $\boldsymbol{B}$ the magnetic field of the electron wave and $\boldsymbol{j}_{\boldsymbol{m}}$ the associated currents, we have:

$$
\partial \boldsymbol{B} / \partial t+4 \pi \boldsymbol{j}_{\boldsymbol{m}}=c \operatorname{curl}(\boldsymbol{E})
$$

For the static radial electric field of an electron at rest, $\operatorname{curl}(\boldsymbol{E})=0$, this does not imply that the magnetic field $\boldsymbol{B}$ and current $\boldsymbol{j}_{\boldsymbol{m}}$, which we assume being the wave of the electron at rest, are null; it means that their sum is zero. If 
the external field is not null, we can identify the right parts of (3) and (4), and using the $k_{i}=\delta \beta / \delta x_{i}$ related solely to the presence of the external field, we have:

$$
\partial \boldsymbol{B} / \partial t+4 \pi \boldsymbol{j}_{\boldsymbol{m}}=\operatorname{curl}\left(2 g_{D}\left(\operatorname{grad}\left(k_{4}\right)-\partial \boldsymbol{\kappa} / \partial t\right)\right)
$$

We obtain the difference between the normal magnetic currents and field of an electron and the currents and fields in the presence of an external static electric field. We can also use the same identification with $(\gamma+\beta)$ and the corresponding $k_{i}=\delta(\gamma+\beta) / \delta x_{i}$ to obtain the same equation as (4) in which the $k_{i}$ include the electron's phase and its variations in space-time. Actually, our understanding of the $k_{i}$ is not quite different from Dirac's, because it is quite tempting to push the identification and to separate the spatial and temporal derivatives in (3), in which case this equation becomes a trivial confirmation of our assumption on the nature of the electron wave. The remainder of (3) is $2 g_{D} \mathrm{curl}(\boldsymbol{k})=\boldsymbol{H}$, which corresponds to $\boldsymbol{B}$ in (4-5), and then the identification goes trivially with $\partial \boldsymbol{B} / \partial t=-2 g_{D} \operatorname{curl}(\partial \boldsymbol{\kappa} / \partial t) \rightarrow \boldsymbol{B}=-2 g_{D} \operatorname{curl}(\boldsymbol{\kappa})+$ const. $=-\boldsymbol{H}+$ const.

This fits with our idea of the electron wave, and partly answers our first remark to Dirac's demonstration, because if the wave of an electron is a magnetic current, the wave of a monopole should be an electric current, resulting in a different symmetry, new constraints in the equations, and a different charge quantum; this is acceptable in theory because (3) comes from knowledge or interpretation of the electron wave, and not from observation of the monopoles.

Let us now look at the singular line, but for an electron; this is quite unusual but it will give a useful picture. The electron field $\boldsymbol{E}$ is defined using a pseudo potential $\boldsymbol{A}$ such that: $\operatorname{curl} \boldsymbol{A}=e \boldsymbol{r} / r^{3}$. Using $\Sigma$ a surface bounded by a loop $\Lambda$, according to Stokes' theorem:

$$
\int_{\Sigma} \boldsymbol{E} \cdot d \boldsymbol{S}=\int_{\Sigma} \operatorname{curl} \boldsymbol{A} \cdot d \boldsymbol{S}=\int_{\Lambda} \boldsymbol{A} \cdot d \boldsymbol{l}=e \int_{\Sigma} \boldsymbol{r} / r^{3} \cdot d \boldsymbol{S}=e \int_{\Sigma} d \Omega
$$

where $d \boldsymbol{S}, d \boldsymbol{l}$ and $d \Omega$ are elements of surface, length and solid angle respectively. Now, if we shrink the loop with the electron inside the closed surface $\Sigma$, we get:

$$
\int_{\Lambda \rightarrow 0} \boldsymbol{A} \cdot d \boldsymbol{l}=e \int_{\Sigma} d \Omega=4 \pi e
$$

But the left integral vanishes, making this equality impossible. The classical solution is a non continuous potential, with a singular line around which the loop shrinks, but this is not acceptable for an electron. A solution to get rid of the singularity is to assume that the integral $\int_{A \rightarrow 0} \boldsymbol{A} \cdot d \boldsymbol{l}=0$ is right, and implies the presence of an isotropic current $\boldsymbol{K}$ verifying:

$$
\int_{\Sigma}(\boldsymbol{E}+\alpha \boldsymbol{K}) \cdot d \boldsymbol{S}=0
$$

The constant $\alpha$ is put in front of $\boldsymbol{K}$ for consistency with the following sections. Using our interpretation of (3), we can naturally define $\boldsymbol{K}=-2 g_{D} \boldsymbol{r} / r^{3} c$, and equation (8) is verified. Now this defines an electron as a source or sink of magnetic current; this is a first solution which is more acceptable than a singularity. A second solution is to see this current as the result of the transformation of a flux of monopoles interacting with the electron. We will find a strong argument to this transformation in the next section and then find part of its geometry.

In this interpretation, the singular line is due to a classical static definition of the field, and if ignored in the equations, the current going thru the surface $\Sigma$ is "brushed out" into a singular line when we shrink the loop - in other words the mathematical representation is appropriate, closer to reality than the usual static $\boldsymbol{E}=-$ grad $U$, and it somewhat requires the electron wave. It then authorizes to compute the relative strengths of the particles charge and wave provided that the right coefficients are put in the equations. But the well known coefficients in (1) are based on known interaction of electric charges, not on experimental knowledge of magnetic poles.

\section{Mass, Wave, Classical Relations}

Using the electron wavelength $\lambda=h / m c$, the de Broglie wave $\left(\lambda_{d}=h / p\right)$ is found as a superposed phase modulation which is the result of relativistic effects. We will denote de Broglie wave when a distinction is needed. We will denote transformation the monopoles interaction with the electron, as opposed to change in phase for the wave interaction with a charge.

Electrons, muons and tauons have the same electric field but their wavelengths are different. The wavelength is not a property of the sole electric field, it is a property of mass and energy, the fact is well known.

An atomic orbital is based on the geometrical constraint that the currents define a stationary wave, a phase shift $2 \pi n$ of the de Broglie wave, but the electron spin imposes $\pi(2 n+1)$ and then the transformation of the current in its interaction with the electron can only correspond to a phase difference $\pi$. This gives quanta of action and of angular momentum thru a unique mechanism, which we can trivially express with the following substitutions:

$$
h=4 \pi e g_{D} / c ; \quad h / 2=e g_{D} / c
$$


The Plank constant has then a clear physical origin as the action of a physical oscillator at work: a phase variation $2 \pi$ of the magnetic currents over one period or wavelength of the electron wave. The quantum of angular momentum is the result of the same mechanism, and relates to the spin of the electron, a phase shift $\pi$ of the current, in agreement with the transformation we supposed in the previous section. Consequently the currents are not made of unitary Dirac charges as their change in phase is not $2 \pi$. Now energy is the frequency of the oscillator, and then there is no doubt on the physical significance of:

$$
E=h v=4 \pi v \text { e } g_{D} / c
$$

There is nothing new in the above equations, except for some notations when we use $g_{D}$ instead of $h$. But the interpretations we give are new, and results from/in coherent circular reasoning from our assumptions, and up to this point, this is what we are looking for: the picture is coherent and provides with a simple and quite classical explanation to some fundamental mysteries of quantum physics.

An important aspect is that the observable energies are not the fundamental quantities, as they are solely the intensity of a magnetic current ( $v g_{D}-$ Dirac charges per second) interacting with the electric charge. This is our trivial answer to the question of the energy source: the concept of energy, as we are used to, is a theoretical preconception, we are often used to think it as a material thing, only because it obeys conservation laws. In fact, we should read literally " $E$ is $h v$ ", in the same manner as momentum is the product of mass and velocity.

Now when the energy of an electron varies, its pulsation changes: $\Delta E=h \Delta v=h c \Delta(1 / \lambda)$. The wavelength of the current varies in reverse proportions, while the electron's electric field remains constant. Thus the fine structure constant is related to the constant of charge $2 g_{D}$ in (3), and concerns the flux of magnetic charges making the electron wave. Our understanding is that in addition to the usual static electric field, a dynamical interaction is at work building the electron mass; this constant is also the coupling of an electron wave with another electric charge thru its wave as $2 g_{D} e \alpha=e^{2}$.

The fine structure constant then applies to the interaction of an electric charge with a magnetic current, and should also, by symmetry, apply to the interaction of a magnetic charge with an electric current, but not to the interaction of charges and currents of identical nature for which coefficients must be trivial. This is why we put the coefficient $\alpha$ in front of the current $K$ in Equation (8).

\section{The Magnetic Monopole}

We shall discuss the changes in the coefficients of (1) in a progressive manner. The first and second sections provide with information needed to complete the quantization using Dirac's method.

\subsection{Phenomenological Charge Quantization}

In our interpretation of the electron wave, monopoles must be massless and have a charge smaller that Dirac's; then we can only study the interaction of a monopole "part of" the wave of an electron; no other option exists.

Let us first look at the symmetry of the problem. Equation (3) provides with the quantitative symmetry of an electron in terms of electric charge and magnetic current. Then denoting $\beta \boldsymbol{X}(r, t)$ the magnetic current in an electron wave, where $\beta$ is a charge and $\boldsymbol{X}(r, t)$ a unitary function, a magnetic charge should be defined by a charge $g$, and an electric current $\xi \boldsymbol{Y}(r, t)$ where $\boldsymbol{Y}(r, t)$ is a possibly different unitary function and $\xi$ an electric charge. The ratios between the charges and the currents should be identical for an electron and a monopole, as the coupling of a charge with the field is proportional to the charge, and coefficients should not depend on the type of charge. This leads to the following equality:

$$
\xi / g=\beta / e \rightarrow g \beta=e \xi
$$

Now as we assume that the wave is made of small monopoles, it is natural that each of them carries momentum. Using classical electromagnetism, the electron will be seen with a fixed electric charge and an oscillating magnetic field; conversely a monopole will be seen with constant magnetic charge and an oscillating electric field. Then denoting $\varphi$ any additional phase difference, the third law of Newton gives:

$$
g \beta \boldsymbol{X}(r, t+\varphi)=-e \xi \boldsymbol{Y}(r, t)
$$

This equation addresses the forces exerted on a monopole "part of" the electron wave, and means that this force, which is $g \beta$, except from some numerical coefficients, is opposed to the classical force exerted by a monopole wave on the electric charge of the electron, which is proportional to $e \xi$, and that the coefficients are the same. Equation (12) implies (11), and then provides with a second approach.

Let us now look at orbitals of the hydrogen atom as the interaction of an electron wave with a proton; $g$ and $\xi$ are unknown, but the change in phase of the monopoles current in their interaction with an electron corresponds to a 
phase shift $\pi$ of the electron wave, as it was previously related to the electron spin. On the other hand, the change in phase of the electron wave around a proton is $\pi(2 n+1)$ for any orbital, and includes one $\pi$ related to the electron spin which is given by the de Broglie phase shift. Over one round trip of the wave, the latter is then $2 \pi n$ and depends on $\alpha e \beta$, and the former gives $\pi$ and depends on $e \xi$, and then:

$$
\alpha e \beta=2 n e \xi
$$

Both parts of this equality are related to phase quantities which can be compared, and the coefficient $\alpha$ is solely on the left hand side in agreement with our understanding of the fine structure constant. This gives an inversion of the coefficients with respect to Dirac's theory and Equation (1): $\alpha$ is in front of $\beta$, and the $2 n$ in front of $\xi$.

Now Equation (13) gives $\xi=\alpha \beta / 2 n$, and then substituting in (11), we have:

$$
g(n)=\alpha e / 2 n
$$

Using $n=3$, we find $g(3)=\alpha$ e/ 6 which is Mikhaïlov estimate of the elementary monopole charge.

By symmetry, the Dirac condition (2) should also apply to monopoles in their interaction with the electron, but using the ratio $\alpha / 2 n$ of (14) and the wave $\xi$ of the monopole; this gives:

$$
e \xi=h c / 2 n \rightarrow \xi=e / 4 n=g / 2 \alpha
$$

Now substituting $\xi$ in (11), we find $\beta=g_{D}$ as expected.

\subsection{Quantization of Self-Energy}

Magnetic poles are usually assumed massive; let us recall that their mass can be estimated from the charge self-interaction $m_{g}=m_{e} g^{2} / e^{2}$, where $m_{e}$ is the electron mass (Barut, 1979; Salam \& Tiomno, 1959; Stephenson, 1957). Our monopole is massless and not energy, and using (11) we should rather write $m_{g}=m_{e} g \xi / e \beta$ as this equation addresses the proportionality of the charge self-interaction; this is equivalent, and we can then continue with mass or energy equations. The contribution of a monopole to the electron mass is proportional to the current it creates; we have $m_{e} c^{2}=h v$, and from (10) we find:

$$
4 \pi \text { e } g v / c=h v g^{2} / e^{2}
$$

This equation reduces to $g=2 \alpha e$, which also reads $e=g / 2 \alpha$, the opposite of Dirac's condition: $g=e / 2 \alpha$. Once again, the coefficients are reversed.

There is no trivial manner to modify this equation and obtain series of charges. The only way seems to impose conditions of phase on the left hand side of (16), in such a way that the transformation of the monopoles is independent of their charge, but done in $n$ periods of the electron pulsation, which is quite similar to our reasoning in the previous section. Then in the left hand side of (16), $v \rightarrow v / n$, and we get:

$$
g(n)=2 \alpha e / n
$$

This equation includes all charges in (14) and then also accepts Mikhaïlov's estimate.

We notice that in (17) the factor 2 is at the numerator while it is at the denominator in (14). In this second approach, we did not use the change in phase $\pi$ of the electron wave and this gives the ratio 4 between (14) and (17); it shows that the change in phase $\pi$ of the electron wave corresponds to changes in phase $\pm \pi / 2$ of individual monopoles of opposite charges. We then need two angles of phase (electron, monopole) to represent the electron state, and $(0,0) \rightarrow(\pi, \pm \pi / 2)$ gives a minimal representation of the transformation. We then need a rotation $4 \pi$ of the phase of the electron wave to get a rotation $2 \pi$ of the monopoles state.

\subsection{Classical Quantization}

In view of the results in $(14-17)$, we understand that Equation (1) addresses the normalized wave function of an electron in electric and magnetic fields created by electric charges, but we now have opposite coefficients for interactions with a magnetic pole. Instead of (1), this gives:

$$
\alpha \operatorname{eurl}(\boldsymbol{k})=\boldsymbol{H}
$$

Now we can use the rule of quantization according to which an electron interacts with a monopole for $n$ periods of its own pulsation to make a change in phase $2 \pi$, from (18) and following Dirac's reasoning, we find series of charges identical to (14) that we shall denote $g(n)$ :

$$
2 \pi / n=\int(\boldsymbol{H}, d \boldsymbol{S}) / \alpha e=4 \pi g / \alpha e \rightarrow g(n)=\alpha e / 2 n
$$

Let us check with the converse situation of a monopole interacting with a fixed electron, which is more relevant for a massless monopole. We must use the same coefficients as in (18), but a monopole interacts $n$ periods of the electron pulsation to make a change in phase $\pm \pi / 2$ of its own wave (or a change in phase $\pi$ of the electron wave). 
It would require $4 n$ times the monopole charge to make a change in phase $2 \pi$ of its own wave; consequently:

$$
(4 n g / \alpha) \operatorname{curl}(\boldsymbol{k})=\boldsymbol{E} \rightarrow(4 n g / \alpha) 2 \pi=\int(\boldsymbol{E}, d \boldsymbol{S})=4 \pi e \rightarrow g(n)=\alpha e / 2 n
$$

Finally, using a complete logic we find appropriate corrections to the coefficients and coherent results according to the symmetry of the problem - which is not trivial at all since the $n$ does not represent the same quantity to get (19) and (20); it actually represents complimentary quantities with opposite symmetries.

This demonstration does not prevent the existence of monopoles with a larger charge, which - using the same coefficients - will give a change in phase $4 \pi n$ of the electron wave instead of $2 \pi / n$ in (19), and leave the electron in its initial state (unlike a change in phase $2 \pi$ ). This leads to a second series that we shall denote $G(n)$ :

$$
4 \pi n=\int(\boldsymbol{H}, d \boldsymbol{S}) / \alpha e=4 \pi G / \alpha e \rightarrow G(n)=n \alpha e
$$

A similar reasoning also applies to (20), and gives the same series (using $2 g$ by symmetry).

We can now trivially interpret the changes in phase $\pi$ and $4 \pi$ in agreement with the interpretation of the wave function as a density of probability of presence, but also in the classical physics approximations with the Poincaré cone: A monopole of charge $\alpha e / 2 n$ is temporarily absorbed (and possibly repelled back) by the electron, while a pole of charge $n \alpha e$ passes thru, and the interaction lasts $n$ or $1 / 2 n$ periods of the electron pulsation respectively; those are, in the classical approximation, related to the number of turns of the monopole spiraling the Poincaré cone (except possibly for some constant numerical coefficients).

\subsection{The Monopole Wave}

Using the same notations as for an electron, the field $\boldsymbol{H}$ of a monopole of charge $g$ is defined by a vector potential $\boldsymbol{A}$ such that: $\operatorname{curl} \boldsymbol{A}=g \boldsymbol{r} / r^{3}$. For a surface $\Sigma$ bounded by a loop $\Lambda$, Stokes' theorem gives:

$$
\int_{\Sigma} \boldsymbol{H} \cdot d \boldsymbol{S}=\int_{\Sigma} \operatorname{curl} \boldsymbol{A} \cdot d \boldsymbol{S}=\int_{\Lambda} \boldsymbol{A} \cdot d \boldsymbol{l}=g \int_{\Sigma} \boldsymbol{r} / r^{3} \cdot d \boldsymbol{S}=g \int_{\Sigma} d \Omega
$$

If we shrink the loop with the pole inside the closed surface $\Sigma$, we get:

$$
\int_{\Lambda \rightarrow 0} \boldsymbol{A} \cdot d \boldsymbol{l}=g \int_{\Sigma} d \Omega=4 \pi g
$$

Of course, Equations $(22-23)$ are almost identical to $(6-7)$; there is no mystery, and following the same reasoning as for the electron, $\int_{\Lambda \rightarrow 0} \boldsymbol{A} \cdot d \boldsymbol{l}=0$ implies a current $\boldsymbol{K}$ verifying:

$$
\int_{\Sigma}(\boldsymbol{H}+\alpha \boldsymbol{K}) \cdot d \boldsymbol{S}=0
$$

The current $K$ is multiplied by $\alpha$, in the same manner as when a current and a charge of different natures interact; then Equation (24) can be seen as the monopole charge self-interaction as for electrons in (8). We then trivially have $\boldsymbol{K}=-g \boldsymbol{r} / \alpha r^{3}$ cancelling the singular line. With respect to the coefficient $2 g_{D}=e / \alpha$ in (3), the monopole wave should be represented with a charge $g / \alpha=2 \xi$ in an equivalent monopole equation, in agreement with (15); consequently, the monopole density of probability of presence is affected by a monopole field according to:

$$
2 \xi \operatorname{curl}(\boldsymbol{k})=\boldsymbol{E} ; \quad 2 \xi\left(\operatorname{grad}\left(k_{4}\right)-\partial \boldsymbol{\kappa} / \partial t\right)=\operatorname{ccurl}(\boldsymbol{A})=\operatorname{cg} \boldsymbol{r} / r^{3}
$$

This equation addresses interactions of monopoles together and shows a perfect symmetry with (3). The equations related to the interaction of magnetic and electric poles together were given in the previous section.

\section{Symmetry in Quantization}

Dirac quantization provides with a mechanism that locks together the quantum of electric and magnetic charges. We saw that this mechanism is not broken, and the one we propose is a little more complete.

a) The main symmetry is in Equation (9), and concerns the proportionality of charges and currents; this is the fundamental symmetry leading to our quantization.

b) Our quantization is essentially the same as Dirac's but uses different parameters in the equations, and we do not impose a change in phase $2 \pi n$ to the electron wave. The symmetry of electromagnetism is the same but we find two suites of magnetic poles obeying opposite progressions.

c) Dirac's quantization coefficients are still valid but relates to the magnetic current of the electron wave. The same methodology is appropriate in all cases.

d) The absence of massive magnetic structures like the atoms we know can easily be explained by the ratio between electric and magnetic charges $\left(e \beta / g \xi \geq(1 / n \alpha)^{2}\right.$ or $\left.(n / \alpha)^{2}\right)$ - what would build the monopoles mass? This reasoning is numerically acceptable but physically wrong or at least incomplete. First, our equations do not prevent the existence of such structures, in particular the existence of monopole pairs. Second, as magnetic poles build the electron's energy, the so called magnetic world is the one we see; it is simply that nature is not redundant and magnetic charges have a specific role. 


\section{Coherence with Experimental Results}

\subsection{Magnetic Charge Detection}

The series of magnetic charges we deduce is consistent with experiments of Ehrenhaft $(1930,1944)$, Schedling (1950), Ferber (1950) and Mikhaïlov $(1982,1985,1987)$ using illuminated ferromagnetic aerosols. This is an important point because as far as we know no theoretical prediction compatible with those charges is found in the literature.

Charges compatible with Dirac's were detected by Mikhaïlov (1991) when the ferromagnetic particle is within a water droplet, but in this experiment, the mass of the particles is increased 6 orders of magnitude. Our interpretation is that Dirac charge is a point of equilibrium, and then in specific low energy situations, a compound Dirac charge can emerge in the form of a current organized by the interaction of monopoles with surrounding matter, but, in our view, not an elementary particle. Then the larger mass of the particles possibly allows a stabilization or longer retention of a large number of poles, up to a point close to equilibrium.

\subsection{Aharonov-Bohm Effect}

The second important experiment was done by Tonomura \& al (1986), and concerns the verification of the Aharonov-Bohm effect. In this experiment, a magnetic field equivalent to a magnetic dipole is created with a small solenoid but the magnetic field is entirely shielded using superconductors. The experiment is usually considered in agreement with Dirac theory, but we believe it better confirms the work in this paper.

The magnetic field of the solenoid is shielded from the electron by a superconductor; there is no doubt about this, but shielding an electron's wave of wavelength $\lambda \approx 2.410^{-12} \mathrm{~m}$ is quite doubtful whatever the method, then the electron wave and currents pass thru the solenoid. When each end of the solenoid is mimicking a Dirac charge the experiment gives an interference pattern corresponding to a change in phase $\pi$ of the electrons wave; this is in agreement with our theory since $2 g_{D}$ is a change in phase $2 \pi$. But according to Dirac (1931) and (2), we should see a change in phase $2 \pi$ with $1 g_{D}$ and then no interference.

The change in phase of our quanta depends on the product $g G$, where $G$ is the charge giving the same magnetic flux as one end of the solenoid. With $G=g_{D}$ and a monopole of charge $g=\alpha e / n$ and current $\xi=e / 2 n$, the change in phase of monopoles is given by $g g_{D}=e^{2 / 2 n}=e \xi$ and corresponds to the same change in phase as for a monopole interacting with an electron: a change in phase $\pi$ of the electron wave. Logically, the coefficients must be the same for $g g_{D}$ and $e \xi$ since both expressions address the interaction of charges and currents of identical nature; then comparing $g G$ with $e \xi$ is appropriate. The other end of the solenoid will give an opposite change in phase $-\pi$ of the electron wave and then, considering elementary monopoles building the wave, it will reinforce the interference. Our theory is then in agreement with this experiment.

\subsection{Hydrogen Atomic Orbitals}

Our initial approach to monopoles charge quantization is equivalent to saying that on orbital $n$, the electron is permanently interacting with monopoles of charge $g(n)=\alpha e / 2 n$ where $n$ is the orbital number. In the non-relativistic case, and using (8), the current of such monopole is directly given by $g(n)$; we have:

$$
E=4 \pi v_{0} \text { e } g(n) / c=4 \pi v_{0} \text { e } g(1) / n c
$$

The only variable term is $n$, and the energy of the orbital is quantized according to the well known $E=E_{0} / n^{2}$. We know precisely the value of $E_{0} \approx 13.6 \mathrm{eV}$ since Bohr's atomic model, and in agreement with quantum physics. Orbitals quantization originally relies on angular momentum, but we now have a direct relation between the electron energy, the orbital number and the monopoles charge in agreement with our understanding of energy.

For orbital number one, we have:

$$
E_{0}=\left(e^{4} m_{e} / 2 h^{2}\right)=\alpha^{2}\left(m_{e} c^{2}\right) / 2=\left(m_{e} c^{2}\right) g(1) / 2 g_{D}
$$

This equation means that the energy loss for the orbital $l$ of a hydrogen atom corresponds to the loss of half the current of one monopole of charge $g(1)=\alpha$ e/2; but on orbital $n$ it will be $g(n) / 2 n=\alpha e / 2 n^{2}$. The additional $1 / 2 n$ represents the number of periods of the electron pulsation (2n) needed for the charge $g(n)$ to come in phase opposition with itself, as this is the basis of our quantization logic to $(20)^{1}$; it is then naturally cancelling, on orbital $n$, once every $2 n$ periods of the electron wave; this gives the expexted energy loss $E_{0} / n^{2}$. ${ }^{\text {1 }}$ (A monopole of charge $g(n)$ interacts $n$ periods of the electron pulsation to make a change in phase $\pm \pi / 2$ of its own wave, and then $2 n$ periods to make a change in phase $\pm \pi$ and come in phase opposition with itself).

A second approach is to consider the charges self-interaction:

$$
m_{e} c^{2} g(n) \xi(n) / e \beta=m_{e} c^{2} g(n) / 2 n g_{D}=E_{0} / 2 n^{2}
$$


This corresponds to only half the energy loss on orbital $n$ - and we should expect twice more; but this is logical as the symmetry between (27) and (28) is the same as for our quantizations to (14) and (17) - on the left hand side of (28), we should actually write $4 g(n)$, as we did in (20), and get twice the energy loss as for (27).

For a muon, the energy $\left(E_{0} \rightarrow E_{0} m_{\mu} / m_{e}\right)$ is different, and (27) is still valid; this shows that the particle frequency cannot be separated from the charge $g_{D}$ in (10), and that only charges and/or frequencies ratios are relevant. (Unfortunately, it also shows that we cannot easily deduce the monopoles velocity and/or wavelength).

For another hydrogenoid with a nucleus of charge $Z e$, the energy loss on orbital $n$ becomes $Z^{2} E_{0} / n^{2}$. One $\mathrm{Z}$ is given by the orbital contraction, but in order to understand the exponent 2 we must go back to (13) which becomes $\alpha(Z e) \beta=2 n e \xi$. The $Z$ has the same role as $1 / n$, and then the rate of cancellation is given by $Z^{2 /} n^{2}$. This point is interesting as it shows that the energy loss due to the cancellation of the current of one monopole can get a value up to $(137)^{2} E_{0} / n^{2}$; with $n=1$, it goes almost up to the full electron's energy, but not more.

This entirely confirms our series of charges $\alpha$ e/2n, with $n>0$, and excludes any other charge smaller that $g(1)$, as they would give unknown orbitals.

\subsection{The Electrons Mass}

Using $E_{0}$ as a fundamental quantity, the electrons' energy is given by $E_{0} / \alpha^{2}=m_{e} c^{2}$. We showed that we cannot split $v g_{D}$ in (10), but it is still interesting to see if we can compute the energy of an electron using a defined sum of monopoles and get an approximate result. A monopole $g(n)$ represents an energy equal to $E_{0} / n$ and $\Sigma 1 / n$ gives a divergent Riemann series. We could stop the analysis at this point and state that the series must end at some different points for elections, muons, and tauons. It diverges so slowly that there is no doubt that we can find the exact mass of any particles with good precision. In our opinion, this would be barely acceptable.

Using simple arithmetic, we find that the following series matches the electron mass:

$$
m_{e} c^{2}=E_{0}(-137+\Sigma(n)) /(137 \alpha)^{2} ; \quad n \text { in } 1 . .274
$$

This is not surprising as it is solely a trivial property of the series $\Sigma n$, but this agrees with (8) as the energy given to the electron by a charge $G(n)$ is $n E_{0}$ which is quite close to $n E_{0} /(137 \alpha)^{2}$. The difference can possibly be understood from the fact that we consider the electron as a phase singularity of its own wave; the difference between $137^{2}$ and $1 / \alpha^{2}$ could come from a spatial extension of the monopoles transformation.

For muons and tauons, Equation (29) is also valid as the corresponding energy given by $E_{0} \rightarrow E_{0} m_{\mu} / m_{e}$ is proportional to the particle mass; this agrees with energy as a current of quantized monopoles and once again it means that the frequency $v$ and the charge $g_{D}$ in Equation (10) cannot be separated.

However, a second approach could be valid; assuming that (29) is a specific case of a more general equation, an empirical search for muons gives:

$$
m_{\mu} c^{2} \approx E_{0}(-137-1970+\Sigma(n)) /(137 \alpha)^{2} ; \quad n \text { in } 1 . .3940
$$

The error is a lack of energy of $60 \mathrm{eV}$ with respect to CODATA (2010) value; to be compared with the muons mass, but also with the last increment of the sum, which is given by $3940 E_{0} \approx 53 \mathrm{KeV}$. For tauons, we find:

$$
m_{\tau} c^{2} \approx E_{0}(-137-8078+\Sigma(n)) /(137 \alpha)^{2} ; \quad n \text { in } 1 . .16156
$$

The error is a lack of energy of $\approx 16 \mathrm{KeV}$ with respect to CODATA (2010) value; the last increment is $\approx 220 \mathrm{KeV}$. The difference is small as compared to the particle mass, but also with respect to the last increment. We do not find an exact match but the error can possibly be related to second order effects or a small non-linearity.

Our assumption on the nature of the electron mass/energy and wave is then compatible with the known electron, muon, and tauon masses-possibly in two different manners.

\section{Conclusions}

Under the sole assumption of the magnetic nature of the electron wave, we showed first that small quantized magnetic charges, including Mikhailov's, are relevant in quantum physics and in agreement with Dirac's quantization method once the coefficients we deduced are put in the equations; we then showed how our understanding of mass/energy works together with charges quantization in agreement with the energy levels of hydrogen atomic orbitals and with the electrons masses. Relevant experimental results related to magnetic poles detection and to Dirac quantization were discussed and found in agreement with the theory we propose. Then, inasmuch as a theory agrees with unexplained repeated experiments and does not conflicts with validated knowledge, it is legitimate to suppose that it brings understanding of the phenomena and mechanisms it describes. Then perhaps we learnt a little about the magnetic poles and the works or origins of the structuring constants of quantum physics. 


\section{References}

Barut, A. O. (1979). Lepton Mass Formula. Physical Review Letters, 42, 1251. http://dx.doi.org/10.1103/PhysRevLett.42.1251

Consiglio, J. (2012/Aa) On particles mass and the Universons hypothesis. Applied Physics Research, 4(2), 144. http://dx.doi.org/10.5539/apr.v4n1p144

Consiglio, J. (2012b/B) Relativity, magnetic charge and weak bosons mass. Applied Physics Research, 4(3), 100. http://dx.doi.org/10.5539/apr.v4n3p100

Curie, P. (1894). Sur la possibilité d'existence du magnétisme libre. J. de Phys., 3(série, III), 415.

Curie, P. (1894). Sur la symétrie dans les phénomènes physiques. J. de Phys., 3(série, III), 393.

De Broglie, L. (1927). Théorie de la double solution. Journal de Physique, 1927.

De Broglie, L. (1987). Interpretation of quantum mechanics by the double solution theory. Ann. Fond. L de Broglie, 12(4). Retrieved from http://aflb.ensmp.fr/AFLB-classiques/aflb124p001.pdf

Dirac, P. A. M. (1931). Quantized singularities in the Electromagnetic Field, Proc. Roy. Soc., A, 133, 60.

Dirac, P. A. M. (1948). The Theory of Magnetic Poles. Phys. Rev., 74, 817. http://dx.doi.org/10.1103/PhysRev.74.817

Dirac, P. A. M. (1978). The Monopole Concept, 17(4), 235.

Ehrenhaft, F. (1930). Die longitudinale und transversale Elektro- und Magnetophorese. Phys. Z., 31, 478.

Ehrenhaft, F. (1944). New Experiments about the Magnetic Current. Physical Review, 65, 62-63.

Ferber, J. A. (1950) Acta Phys, Australica, 4(1950), 133w.

Lochak, G. (1995). The Symmetry between Electricity and Magnetism, Advanced Electromagnetism. World Scientific, Singapore. Retrieved from http://aflb.ensmp.fr/MEMOS/GLmonopole/MONOPOLE.pdf

Lochak, G. (2007). The symmetry between Electricity and Magnetism and the equation of a leptonic Monopole Fondation Louis de Broglie. Retrieved from http://arxiv.org/abs/0801.2752

Maxwell, J. C. (1873). A Treatise on Electricity and Magnetism (3rd ed.). Clarendon Press, Dover 1954.

Mikhaïlov, V. F. (1982). Physical Review Letters, 1303, 331.

Mikhaïlov, V. F. (1985). Observation of magnetic monopoles in the field of a line conductor with a current. $J$. Phys. A, 18, L903. http://dx.doi.org/10.1088/0305-4470/18/14/014

Mikhaïlov, V. F. (1987). Light microparticles and magnetic charge phenomenon. Ann. Fond. L. de Broglie, 12, 491.

Mikhaïlov, V. F. (1991). Observation of apparent magnetic charges carried by ferromagnetic particles in water droplets. J. Phys. A, 24, 53. http://dx.doi.org/10.1088/0305-4470/24/1/016

Mikhaïlov, V. F. (1993). Courants, Amers, Ecueils en Microphysique. Fond. L. de Broglie, Paris, 1993.

Salam, A., \& Tiomno, J. (1959). On the masses of elementary particles. Nuclear Physics, 9, 585. http://dx.doi.org/10.1016/0029-5582(58)90341-9

Schedling, J. A. (1950). Acta Phys. Australica, 4, 98.

Stephenson, G. (1957). A classical calculation of the nucleon-meson coupling costant. Nuovo Cimento, 5, 1009. http://dx.doi.org/10.1007/BF02903225

Tonomura, A. et al. (1986) Evidence for Aharonov-Bohm effect with magnetic field completely shielded from electron wave. Phys. Rev. Lett., 56, 792-795. http://dx.doi.org/10.1103/PhysRevLett.56.792 